\title{
Vacuum expectation value renormalization in the standard model and beyond
}

\author{
Vytautas Dūdènas $\odot^{*}$ \\ Institute of Theoretical Physics and Astronomy, Faculty of Physics, Vilnius University, \\ 9 Sauletekio, LT-10222 Vilnius, Lithuania \\ Maximilian Löschner® ${ }^{\dagger}$ \\ Institute for Theoretical Physics, Karlsruhe Institute for Technology, \\ Wolfgang-Gaede-Straße 1, 76131 Karlsruhe, Germany
}

(Received 29 October 2020; accepted 8 March 2021; published 13 April 2021)

\begin{abstract}
We show how the renormalization constant of the Higgs vacuum expectation value fixed by a tadpole condition is responsible for gauge dependences in various definitions of parameters in the $R_{\xi}$-gauge. Then we show the relationship of this renormalization constant to the Fleischer-Jegerlehner (FJ) scheme, which is used to avoid these gauge dependences. In this way, we also present a viewpoint on the FJ scheme complementary to the ones already existing in the literature. Additionally, we compare and discuss different approaches to the renormalization of tadpoles by identifying the similarities and relations between them. The relationship to the Higgs background-field renormalization is also discussed.
\end{abstract}

DOI: 10.1103/PhysRevD.103.076010

\section{INTRODUCTION}

In modern particle physics, high precision calculations are of increasing importance for finding signs of new physics in the comparisons of theory predictions to experimental data. An integral part of these calculations is the subject of renormalization. Even though the main principles of renormalization are well understood (see, e.g., [1] for a recent review of electroweak radiative corrections) and represent a standard textbook subject, some subtleties are still actively discussed to the present day. One of these is the subject of vacuum expectation value (VEV) renormalization in conjunction with so-called tadpole schemes. For existing examples of discussions in the literature, see, e.g., [2-8] or [1] for a list of tadpole schemes. However, we find that a unified exposition of the relationships between such schemes is still missing in the literature. Hence, in this paper, we want to elucidate the relation between the renormalization of vacuum expectation values, tadpole schemes, gauge dependences, and the special role of Goldstone boson tadpoles in this respect. More specifically, we show the connections between

\footnotetext{
*vytautasdudenas@inbox.lt

maximilian.loeschner@kit.edu
}

Published by the American Physical Society under the terms of the Creative Commons Attribution 4.0 International license. Further distribution of this work must maintain attribution to the author(s) and the published article's title, journal citation, and DOI. Funded by SCOAP. methods that are commonly used in precision calculations for the Standard Model (SM), as, e.g., in [1] and more formal discussions of VEV renormalization in general gauge theories as, e.g., in $[9,10]$.

We want to emphasize the known fact that an independent VEV (or tadpole) renormalization constant is necessary in addition to the renormalization of the parameters and fields of the unbroken theory in order to render all $n$-point Green's functions finite in the $R_{\xi}$-gauge (this was already noted in, e.g., $[11,12])$. In the broken phase, the usage of the VEV in gauge-fixing functions affects the global symmetry properties of the theory [9], which leads to the need of this additional degree of freedom. ${ }^{1}$ Hence, in spontaneously broken gauge theories such as the SM, this can be understood as an artifact of the gauge-fixing procedure rather than a direct consequence of the mechanism of spontaneous symmetry breaking itself. This introduction affects the definitions of the parameters, leading to gauge dependences in some of them. In principle, these gauge dependences will always cancel in physical observables. Moreover, the S-matrix can even be made finite without renormalizing tadpoles at all, hence, leaving the one-point Green's functions infinite [11]. Nevertheless, it can be favorable to demand gauge-independent physical parameter definitions in perturbative calculations, i.e., for

\footnotetext{
${ }^{1}$ Note that in some older literature, when the $R_{\xi}$-gauge was not as commonly used as it is nowadays, this fact might not be mentioned. As an example, in [13-15], this fact is not discussed due to the use of $R$-gauge fixing.
} 
intermediate expressions such as mass counterterms. The Fleischer-Jegerlehner tadpole scheme (FJ scheme) [16] was proposed to avoid these spurious gauge dependences. This becomes even more important if one goes beyond the Standard Model (BSM), where the usual on-shell (OS) renormalization is not possible for all parameters (see [17] for a discussion of the problems arising here) and explains part of the renewed attention to the subject in the context of the two-Higgs-doublet models $[5-8,18]$.

The FJ scheme is closely related to the aforementioned additional independent VEV-renormalization constant. As we will see, the FJ scheme makes sure that this degree of freedom will not enter the parameter and counterterm definitions, and hence, allows for gauge-independent definitions. However, neither in the original paper, nor in the more recent ones on the scheme, is this relationship explicitly exposed. Instead, the notions of the "proper VEV" [16] or the "correct one-loop minimum" $[1,5,8,18]$ are used to motivate the gauge cancellations in the FJ scheme. We try to fill the gap by exposing this feature and also suggest looking at the scheme as simply being a convenient set of counterterm redefinitions. Using this viewpoint, we also show translations between different tadpoles schemes.

With a pedagogical purpose in mind, we carry out our study for the SM at the one-loop level, but also comment on the implications for BSM. Moreover, the paper is set up such that our results can be easily reproduced using, e.g., the native FeynArts [19] SM file together with Feyncalc [2022] or FormCalc $[23,24]$ (both with and without background fields).

Note that in order to extend our discussion of gauge dependences to higher-loop orders, one has to adopt the complex mass scheme (CMS) [25-31] in addition to using the FJ scheme. The reason being that in the presence of unstable particles, propagator poles can acquire imaginary parts from two loops onward, and the usual OS scheme leads to gauge-dependent mass definitions in that case. One can therefore only prove the gauge independence of the complex propagator poles [27] and needs to include this in the discussion via the CMS.

We start our presentation in Sec. II by explaining why it is necessary to have an additional renormalization constant in the spontaneously broken phase of the SM as compared to the unbroken phase and introduce a tadpole condition to fix the former. In Sec. III, we present the translations between the renormalization constants of different parameter sets that are used as independent in the renormalization procedure. In particular, we show the relations between renormalization constants of symmetry-based (or "original") parameters of the theory to the ones used in the usual OS scheme in [32]. These translations illuminate the gauge dependences in the definitions of the usual mass renormalization constants. In Sec. IV, we define the FJ scheme as known from the literature, show how it provides gaugeindependent counterterm definitions, and present a new viewpoint on the scheme in terms of renormalization constant reparametrization. This section also relates the FJ scheme to the findings presented in [9], and moreover, we illustrate the differences between the tadpole schemes by comparing VEV-renormalization constants numerically and comment on the outcomes. We conclude our presentation in Sec. V. Some details of our calculations can be found in the appendixes. These include a short note on the construction of the $R_{\xi}$-gauge in the background-field formalism in Appendix A, the calculation of the purely gauge-dependent divergences using the background fields in the SM (an adaptation from [9]) in Appendix B, consequences of different renormalization conditions of this approach in Appendix C, explicit divergences of renormalization constants in Appendix D, and numerical input values that we used to calculate VEV-renormalization constants in Appendix E.

\section{AN ADDITIONAL COUNTERTERM IN $\boldsymbol{R}_{\xi}$}

The necessity of an independent VEV-renormalization constant for renormalizing one-point Green's functions has been noted before (e.g., see [11,12,33]). However, one might come to the conclusion that this is done purely for convenience. The reasons for this impression are given by the fact that S-matrix elements are finite even without renormalizing tadpoles at all [11] or the fact that a gauge fixing other than the $R_{\xi}$-gauge is used, such as in [13-15], where indeed all $n$-point Green's functions can be made finite via multiplicative renormalization of the parameters of the unbroken theory. Here, we want to clarify that the latter statement is not true in the $R_{\xi}$-gauge. As discussed rather recently in [9], the explanation comes from the fact that this gauge fixing explicitly breaks a global $S U(2) \times U(1)$ symmetry. Then, a VEV counterterm cannot be forbidden on the grounds of symmetry arguments, or in other words, its divergence structure is not fixed by the field strength renormalization of a physical scalar. In order to fix its divergence structure, the authors of [9] restore the original global symmetries of the theory by the introduction of background fields ${ }^{2}$ and express the VEV renormalization in terms of the background-field renormalization. Additionally, via the use of Becchi-RouetStora-Tyutin (BRST) sources, the difference between the VEV's divergence structure and the physical scalar field's renormalization is isolated. We will now clarify these points explicitly for the SM.

We consider the usual Higgs potential of the SM:

$$
V(\phi)=\mu^{2} \phi^{\dagger} \phi+\lambda\left(\phi^{\dagger} \phi\right)^{2} .
$$

The neutral component of the Higgs doublet $\phi$ acquires a VEV $v$ as in

\footnotetext{
${ }^{2}$ This setup is also related to more formal studies of algebraic renormalization [34-39]. In these studies, the term rigid symmetry is used instead of global symmetry, but the meaning is the same.
} 


$$
\phi=\left(\begin{array}{c}
G_{W}^{+} \\
\frac{1}{\sqrt{2}}\left(v+h+i G_{Z}\right)
\end{array}\right)
$$

where $G_{W}^{+}$and $G_{Z}$ are the Goldstone boson fields and $h$ is the physical Higgs field. The tree-level minimum condition for Eq. (1) gives

$$
\left.\frac{\partial V}{\partial h}\right|_{h=G_{W / Z}=0}=0 \Rightarrow v^{2}=-\frac{\mu^{2}}{\lambda},
$$

which leads to the Higgs mass being

$$
m_{h}^{2}=2 \lambda v^{2}=-2 \mu^{2} .
$$

Following [9], we introduce background fields denoted by "hats" via

$\phi \rightarrow \phi+\hat{\phi}=\left(\begin{array}{c}G_{W} \\ \frac{1}{\sqrt{2}}\left(h+i G_{Z}\right)\end{array}\right)+\left(\begin{array}{c}\hat{G}_{W}^{+} \\ \frac{1}{\sqrt{2}}\left(v+\hat{h}+i \hat{G}_{Z}\right)\end{array}\right)$.

The $R_{\xi}$-gauge-fixing function is modified in such a way that the gauge fixing and ghost part of the Lagrangian are invariant under the global gauge transformation, where the gauge parameters are restricted to ${ }^{3}$

$$
\xi=\xi_{W}=\xi_{Z}=\xi_{A}
$$

An explicit construction of the gauge-fixing functions using background fields in the SM is explained in Appendix A. In this case, one finds that all $n$-point Green's functions can be rendered finite using only multiplicative renormalization constants,

$$
p \rightarrow Z_{p} p, \quad f \rightarrow \sqrt{Z_{f}} f, \quad \hat{f} \rightarrow \sqrt{Z_{\hat{f}}} \hat{f}
$$

for all parameters $p$, fields $f$, and background fields $\hat{f}$ of the theory. However, in order to isolate purely gaugedependent divergences ${ }^{4}$ in a single constant $\hat{Z}_{\phi}$, we write the field and background-field renormalization as

\footnotetext{
${ }^{3}$ An explicit calculation shows that at one loop, it is enough to use a single additional counterterm even without the equality of the gauge parameters of Eq. (6). However, it is unclear whether this holds at higher-loop orders. One can construct a gauge-fixing function which preserves the global invariance even when Eq. (6) does not hold instead. Such a gauge-fixing function was introduced in [39], yet we want to focus our discussion on the widely used $R_{\xi}$-gauge.

${ }^{4}$ Note that $\bar{Z}_{\phi}$ can nevertheless have gauge-dependent finite parts. This effectively comes about due to the Passarino-Veltmann function $B_{0}\left(p^{2}, m^{2} \xi, m^{2} \xi\right)$ carrying gauge-independent UV divergent but gauge-dependent finite terms. Hence, $\bar{Z}_{\phi}$ can just be used as a tool to disentangle divergence structures.
}

$$
\phi+\hat{\phi} \rightarrow \sqrt{\bar{Z}_{\phi}}\left(\frac{1}{\sqrt{\hat{Z}_{\phi}}} \phi+\sqrt{\hat{Z}_{\phi}} \hat{\phi}\right)
$$

and identify the renormalization of the components of the physical scalar and VEV by

$$
\begin{aligned}
\sqrt{Z_{h}} & =\sqrt{\bar{Z}_{\phi} / \hat{Z}_{\phi}}, \\
Z_{v} & =\sqrt{Z_{\hat{h}}}=\sqrt{\bar{Z}_{\phi} \hat{Z}_{\phi}} .
\end{aligned}
$$

It is now comfortable to introduce, schematically,

$$
\delta=Z-1
$$

for every renormalization constant. Then from Eqs. (9a) and $(9 b)$ at one loop we have $e^{5}$

$$
\delta_{h}=\bar{\delta}_{\phi}-\hat{\delta}_{\phi}, \quad \delta_{v}=\frac{1}{2}\left(\bar{\delta}_{\phi}+\hat{\delta}_{\phi}\right)
$$

It is thus clear that the VEV renormalization can coincide with the Higgs field renormalization only if $\hat{\delta}_{\phi}$ vanishes. We show how one can get the divergent part of $\hat{\delta}_{\phi}$ with the help of BRST sources in Appendix. B. The result for the UV divergent part is

$$
\left.\hat{\delta}_{\phi}\right|_{\mathrm{UV}}=\frac{2}{4-D} \frac{\xi}{16 \pi^{2} v^{2}}\left(2 m_{W}^{2}+m_{Z}^{2}\right)
$$

where $D$ is the number of space-time dimensions, and $m_{W}$ and $m_{Z}$ are masses of $W$ and $Z$ bosons, respectively. Hence, it clearly vanishes for $\xi \rightarrow 0$, and can only then let the VEV renormalization coincide with the Higgs field renormalization.

To further see the role of $\hat{\delta}_{\phi}$, we consider the Higgs onepoint function. By inserting renormalization constants into Eq. (1), collecting all the terms linear in $h$, and using the tree-level minimum condition Eq. (3), we get a counterterm for the one-point function of $h$, i.e.,

$$
\delta t_{h}=-\lambda v^{3}\left(\delta_{\lambda}-\delta_{\mu^{2}}+\bar{\delta}_{\phi}+\hat{\delta}_{\phi}\right),
$$

\footnotetext{
${ }^{5}$ Note that since we started with the multiplicative constants, all parameter renormalization constants including the one of the VEV are defined dimensionless, leading to simpler relations between the constants. The translation to the dimensionful constants can be easily done by replacing

$$
\delta_{p} \rightarrow \frac{\delta p}{p}
$$

in all our expressions.
} 
which is fixed by the tadpole condition, i.e.,

$$
\delta t_{h}+T_{h}=0,
$$

where $T_{h}$ are the one-loop tadpole contributions. Throughout the rest of this paper, we keep this as a fixed condition in all different tadpole schemes. The gaugedependent part of the tadpole function $T_{h}$ is

$$
\begin{aligned}
& T_{h}^{(\xi)}=\begin{array}{c}
G^{ \pm} \\
1 \\
\vdots \\
i
\end{array} \\
& =\frac{1}{16 \pi^{2}} \frac{2 \lambda v^{2}}{v}\left[A_{0}\left(\xi m_{W}^{2}\right)+\frac{1}{2} A_{0}\left(\xi m_{Z}^{2}\right)\right] \text {, }
\end{aligned}
$$

where $(\xi)$ denotes that we take only the gauge-dependent tadpoles, and $A_{0}$ is a one-point Passarino-Veltman function [40]. Checking the UV divergences in Eq. (16) and using Eq. (14) in Eq. (15), we see that the gauge-dependent divergences cancel as

$$
\left.T_{h}^{(\xi)}\right|_{\mathrm{UV}}-\left.\lambda v^{3} \hat{\delta}_{\phi}\right|_{\mathrm{UV}}=0
$$

hence, $\hat{\delta}_{\phi}$ alone absorbs all the gauge-dependent divergences in the tadpole condition. As shown in [7] for the multiHiggs-doublet SM, one can come to the same conclusions by simply demanding the finiteness of all scalar $n$-point functions via $\left\{\delta_{\mu^{2}}, \delta_{\lambda}, \hat{\delta}_{\phi}, \delta_{h}\right\}$, where $\hat{\delta}_{\phi}$ is introduced ad hoc as an additional VEV-renormalization constant and without any reference to the background-field formalism. ${ }^{6}$ For a derivation of this kind in the SM, we show the divergences of the relevant $n$-point functions in Appendix D.

When using the $R_{\xi}$-gauge fixing in the $\mathrm{SM}$, there are no gauge-dependent divergences in other renormalization constants (see Appendix D for explicit expressions); hence, we have

$$
\left.\frac{\partial}{\partial \xi} \delta_{\lambda}\right|_{\mathrm{UV}}=\left.\frac{\partial}{\partial \xi} \delta_{\mu^{2}}\right|_{\mathrm{UV}}=\left.\frac{\partial}{\partial \xi} \bar{\delta}_{\phi}\right|_{\mathrm{UV}}=0
$$

Since $\left.\hat{\delta}_{\phi}\right|_{\text {UV }}$ vanishes when $\xi \rightarrow 0$, while the gauge-independent part is obviously untouched by this limit, we can conclude that $\hat{\delta}_{\phi}$ renormalizes purely the spurious divergences that are caused the gauge-fixing procedure in the $R_{\xi}$-gauge. It is therefore only necessary as an independent renormalization constant when $\xi \neq 0$. In this sense, it is the minimal addition to the set of renormalization parameters of the unbroken theory in order to render all $n$-point Green's functions finite. Moreover, any inclusion of $\hat{\delta}_{\phi}$ in counterterm definitions will carry over its gauge

\footnotetext{
${ }^{6}$ In [7], $\delta v_{k}$ is the equivalent of $v \hat{\delta}_{\phi}$.
}

dependence. As we will see in the next section, this is usually the case for mass counterterms.

\section{A. Remarks}

Employing the tadpole condition of Eq. (15) is of special interest when working with the generating functional for the one particle irreducible (1PI) Green's functions, which is defined as the Legendre transformation of the generating functional for the connected Green's functions. This transformation is only well defined for vanishing one-point Green's functions, making Eq. (15) essential [27,41,42]. The 1PI generating functional in turn is used for deriving functional identities such as the Slavnov-Taylor [43] or Nielsen identities [44].

Questions about gauge dependence in connection with VEV renormalization can also be very relevant in studies of effective loop potentials. It has already been noted in [45], in the context of an Abelian-Higgs model, that Goldstone boson tadpoles violate the so-called Higgs-low-energy theorem (HLET). This relates to an older finding that one can not get Goldstone boson tadpoles from any potential by taking a derivative with respect to the Higgs VEV [3]. The violation of the HLET is in correspondence to the necessity of $\hat{\delta}_{\phi}$ in the $R_{\xi}$-gauge. In [45], the $R_{\xi}$-gauge is traded for the so-called $R_{\xi, \sigma}$-gauge, which reinstates a global symmetry of the Lagrangian and in this way avoids the necessity of an independent VEV counterterm though.

Similarly, in studies of finite temperature phase transitions (see, e.g., [46,47]), one finds gauge-dependent positions of the minima of effective loop potentials. ${ }^{7}$ Here, this is a result of using an $R_{\xi}$-gauge and defining the effective action as the sum of 1PI graphs, i.e., without tadpole and other external leg contributions. Moreover, it is interesting to find the diagram in Fig. 1 of [47], which determines the Nielsen coefficient of a one-loop effective potential. Our definition of $\hat{\delta}_{\phi}$ via BRST sources shown in Fig. 2 of Appendix B is the equivalent of this in terms of its divergence structure.

As a last note in this section, we would like to stress the differences in the use of background fields in $[9,48]$. It might appear as if the authors state exact opposites, namely, that a nonzero VEV counterterm is strictly necessary versus the statement that no VEV renormalization in addition to the Higgs field renormalization is needed. ${ }^{8}$ However, both statements are not contradictory, as the respective contexts differ. In [48], the authors do not renormalize quantum fields at all, as they are interested only in the Green's functions of the background fields. Then, the statement that no genuine VEV counterterm is needed translates to the fact that no

\footnotetext{
${ }^{7}$ Nevertheless, the values of the potentials at these points are found to be gauge independent.

${ }^{8}$ Both references use the notation $\delta v$ for different quantities leading to potential confusion.
} 
renormalization in addition to Eq. (9b) is necessary. In [9], however, the focus lies on the renormalization of quantum fields, while the background fields are still used to preserve the symmetry structure of the theory. Then, $\hat{Z}_{\phi}$ in Eq. (9b) is interpreted as an additional counterterm to the one of Eq. (9a), due to a mismatch between quantum field renormalization and the renormalization of its VEV. In their notation, $\delta v \neq 0$ as they parametrized it in a relationship with the quantum instead of the background-field renormalization as compared to [48].

\section{TRANSLATION OF RENORMALIZATION CONSTANTS}

Before a renormalization procedure is carried out, one has to choose a set of independent renormalization constants. In the SM, one usually chooses experimentally well-accessible physical parameters as independent renormalization constants, while in BSM studies, it can be convenient to use the set of original theory parameters and the VEVs, especially when the use of an $\overline{\mathrm{MS}}$ scheme cannot be avoided. For the comparability of different choices, it is instructive to have a translation between the sets of renormalization constants. To get these relations, consider that we have a parameter set $\{p\}$ related at tree level to a parameter set $\left\{p^{\prime}\right\}$ by some function $f$ :

$$
p_{i}^{\prime}=f_{i}(\{p\}) .
$$

Introducing renormalization constants as in Eq. (B9) and expanding to one-loop order induces the relations

$$
\delta_{p_{i}^{\prime}}=\frac{1}{f_{i}(\{p\})} \cdot\left(\delta_{p_{j}} p_{j}\right) \frac{\partial}{\partial p_{j}} f(\{p\}) .
$$

In the SM, the relevant set of tree-level relations is

$$
\begin{aligned}
m_{h}^{2} & =\mu^{2}+3 \lambda v^{2}, & m_{W} & =\frac{v}{2} g_{2}, \quad m_{Z}=\frac{v}{2} \sqrt{g_{1}^{2}+g_{2}^{2}}, \\
e & =\frac{g_{1} g_{2}}{\sqrt{g_{1}^{2}+g_{2}^{2}}}, & t_{h} & =-v\left(\mu^{2}+v^{2} \lambda\right) .
\end{aligned}
$$

Using Eq. (20), we get the relations between the renormalization constants of the parameter renormalized tadpole scheme (PRTS) [32], usually used with an OS scheme, to the ones of the original parameters, namely,

$$
\left\{t_{h}, m_{h}, m_{W}, m_{Z}, e\right\} \leftrightarrow\left\{v, \mu^{2}, \lambda, g_{1}, g_{2}\right\},
$$

where $g_{1}$ and $g_{2}$ are the $U(1)$ - and $S U(2)$-couplings, respectively. The results are shown in the first column of Table I, where $\delta_{v}$ is expressed in terms of the field renormalization constants of Eq. (12). This is to show where the gauge-fixing-induced $\xi$-dependences appear via $\hat{\delta}_{\phi}$. Inspecting the first column of Table I, one clearly sees that $\hat{\delta}_{\phi}$ enters the definition of the usual mass counterterms. This means that the latter are necessarily gauge dependent if one defines them as in the PRTS. In the next section, we will present the FJ scheme and show its role in the cancellation of gauge dependences by virtue of the renormalization constant redefinitions shown in the second column of Table I.

\section{RELATIONS AMONG DIFFERENT VEV SCHEMES}

\section{A. FJ scheme}

The FJ scheme is a procedure of reinstating tadpole contributions in perturbative calculations so that parameter definitions can be defined in a gauge-independent way.

TABLE I. The mass, VEV, and electric charge renormalization constants are expressed in terms of the renormalization constants of gauge couplings $g_{1}, g_{2}$ and potential parameters $\lambda, \mu$ together with the (background) field renormalization constants in the two tadpole schemes. This is to emphasize the relations of divergence structures between the different renormalization constants. $\Delta$ is the "FJ term" used to relate counterterms from the usual tadpole scheme to the FJ scheme (see Sec. IVA).

\begin{tabular}{lc}
\hline \hline Usual tadpole scheme [32] & FJ scheme [16] \\
\hline$\Delta=0$ & $\Delta=\frac{T_{h}}{v m_{h}^{2}}=\frac{1}{2}\left(\delta_{\lambda}-\delta_{\mu^{2}}+\bar{\delta}_{\phi}+\hat{\delta}_{\phi}\right)$ \\
$\delta_{v}=\frac{1}{2}\left(\bar{\delta}_{\phi}+\hat{\delta}_{\phi}\right)$ & $\left.\delta_{v}\right|_{\mathrm{FJ}}=\frac{1}{2}\left(\delta_{\mu^{2}}-\delta_{\lambda}\right)$ \\
$\delta t_{h}=-\lambda v^{3}\left(\delta_{\lambda}-\delta_{\mu^{2}}+\bar{\delta}_{\phi}+\hat{\delta}_{\phi}\right)$ & $\left.\delta t_{h}\right|_{\mathrm{FJ}}=0$ \\
$\delta_{M_{h}^{2}}=\frac{3}{2}\left(\delta_{\lambda}+\bar{\delta}_{\phi}+\hat{\delta}_{\phi}\right)-\frac{1}{2} \delta_{\mu^{2}}$ & $\left.\delta_{M_{H}^{2}}\right|_{\mathrm{FJ}}=\delta_{\mu^{2}}$ \\
$\delta_{M_{W}^{2}}=2 \delta_{g_{2}}+\bar{\delta}_{\phi}+\hat{\delta}_{\phi}$ & $\left.\delta_{M_{W}^{2}}\right|_{\mathrm{FJ}}=2 \delta_{g_{2}}+\delta_{\mu^{2}}-\delta_{\lambda}$ \\
$\delta_{M_{Z}^{2}}=2 \frac{g_{2}^{2} \delta_{g_{2}}+g_{1}^{2} \delta_{g_{1}}}{g_{1}^{2}+g_{2}^{2}}+\bar{\delta}_{\phi}+\hat{\delta}_{\phi}$ & $\left.\delta_{M_{Z}^{2}}\right|_{\mathrm{FJ}}=2 \frac{g_{2}^{2} \delta_{g_{2}}+g_{1}^{2} \delta_{g_{1}}}{g_{1}^{2}+g_{2}^{2}}+\delta_{\mu^{2}}-\delta_{\lambda}$ \\
$\delta_{m_{f}}=\delta_{y}+\frac{1}{2}\left(\bar{\delta}_{\phi}+\hat{\delta}_{\phi}\right)$ & $\left.\delta_{m_{f}}\right|_{\mathrm{FJ}}=\delta_{y}+\frac{1}{2}\left(\delta_{\mu^{2}}-\delta_{\lambda}\right)$ \\
& \\
\hline \hline
\end{tabular}


In the current literature, this procedure is often paraphrased as a shift of the VEV to the correct minimum of the loop-corrected scalar potential $[1,5,8,18]$. This means that one first shifts the bare VEV by the full tadpole contributions, i.e.,

$$
v_{\text {bare }}=\left.v_{\text {bare }}\right|_{\mathrm{FJ}}+\Delta v, \quad \Delta v=\frac{T_{h}}{m_{h}^{2}},
$$

where we indicated the shifted VEV by "FJ." Only then, one adopts multiplicative renormalization constants and inserts $\Delta v$ at each appearance of the VEV in the Lagrangian before using the parameter relations of Eq. (21). Since Eq. (23) constitutes a redefinition of the bare VEV, it no longer can be interpreted in terms of the background-field renormalization constant, and we introduce an independent $\left.\delta_{v}\right|_{\mathrm{FJ}}$ for its renormalization. Then, the initial bare VEV is related to the renormalized VEV $v$ as

$$
v_{\text {bare }}=v+\left.v \delta_{v}\right|_{\mathrm{FJ}}+\Delta v .
$$

The full tadpole counterterm becomes

$$
\delta t_{h}=-\lambda v^{3}\left(\delta_{\lambda}-\delta_{\mu^{2}}+\left.2 \delta_{v}\right|_{\mathrm{FJ}}\right)-\Delta v m_{h}^{2} .
$$

Using the definition of the FJ-VEV shift, Eq. (23), we see that the first term of Eq. (25) must vanish in order to fulfill the tadpole condition of Eq. (15), which leads to the identification

$$
\left.\delta_{v}\right|_{\mathrm{FJ}}=\frac{1}{2}\left(\delta_{\mu^{2}}-\delta_{\lambda}\right)
$$

This shows that in the FJ scheme, one recovers the treelevel relation between the VEV and the parameters of the scalar potential of Eq. (3), so that the VEV renormalization can be fully expressed in terms of shifts of the potential parameters $\mu^{2}$ and $\lambda$. In this sense, the shift of Eq. (23) can be paraphrased as shifting the VEV to the correct one-loop minimum. Or, as presented in [16], one chooses the proper VEV.

Introducing the shift of Eq. (23) into the Lagrangian everywhere is effectively equivalent to including regular tadpole contributions in the Green's and vertex functions in addition to the 1PI contributions, i.e., effectively setting $\delta t_{h}=0$ as well as $\Delta=0$. Nevertheless, the FJ scheme has the appeal that renormalized one-point functions vanish exactly with the tadpole condition Eq. (15) being fulfilled, while they would remain formally divergent in the latter case. Since Eq. (26) does not include $\hat{\delta}_{\phi}$ in its definition, it has no gauge-dependent UV divergences in contrast to the PRTS. This is shown in Table I where we also see that none of the other parameters carries a $\hat{\delta}_{\phi}$-dependence in the FJ scheme. Here, we see the relationship between the FJ scheme and the additional VEV-renormalization constant of [9], or equivalently, $\hat{\delta}_{\phi}$ : The FJ scheme cancels the gauge-dependent divergences which come about due to the breaking of the global gauge symmetry via the $R_{\xi}$-gauge fixing. We will discuss the gauge dependence of finite parts at the end of the section.

\section{B. FJ reparametrization}

Equation (23) together with the tadpole condition Eq. (15) might seem as two renormalization steps. However, Eq. (23) represents a mere redefinition of a bare parameter. In the following, we make this redefinition more explicit and thereby give an equivalent way of formulating the FJ scheme. This will also simplify the comparison of the different tadpole schemes.

We first generate counterterms via multiplicative renormalization of the parameters $\left\{v, \mu^{2}, \lambda, g_{1}, g_{2}\right\}$ and then do a simple zero insertion of what we call a $F J$ term $\Delta$ in the bare VEV, i.e.,

$$
v_{\text {bare }}=v\left(1+\delta_{v}-\Delta+\Delta\right),
$$

where $\Delta$ is the dimensionless equivalent of the VEV shifts used in Eq. (23), namely,

$$
\Delta=\frac{T_{h}}{v m_{h}^{2}}
$$

We then redefine the VEV counterterm by

$$
\left.\delta_{v}\right|_{\mathrm{FJ}}=\delta_{v}-\Delta,
$$

which gets us back Eq. (24), and thereby make the relation between the two tadpole schemes explicit. Similarly, we can identify

$$
\begin{aligned}
& \left.\delta_{m_{f}}\right|_{F J}=\delta_{m}-\Delta, \\
& \left.\delta_{m_{V}^{2}}\right|_{F J}=\delta_{m_{V}^{2}}-2 \Delta, \\
& \left.\delta_{m_{h}^{2}}\right|_{F J}=\delta_{m_{h}^{2}}-3 \Delta,
\end{aligned}
$$

and thereby parametrize the change in going from the renormalization constants of the PRTS to the ones of the FJ scheme. In deriving the last line of Eq. (30c), care needs to be taken by imposing the tadpole condition only after the shift Eq. (29) was inserted.

Diagrammatically, Eq. (27) can be understood as a simple reassignment of the two instances of $\Delta$. In the case of one-loop corrections to a fermion mass, this means 


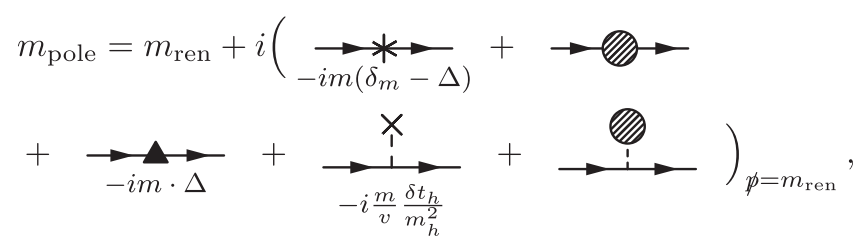

where one can identify the FJ mass renormalization constant of Eq. (30a) in the first diagram, which has no gauge-dependent divergences due to the cancellation of $\hat{\delta}_{\phi}$ in its definition, while the third diagram represents implicit tadpole contributions. Instead, if we choose $\Delta=0$, the first diagram represents a gauge-dependent mass counterterm $\delta_{m}$ as defined in the PRTS. In any case, the usual tadpole condition of Eq. (15) provokes the cancellation of the last two diagrams.

Generically, we can write

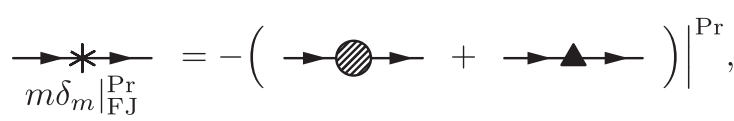

in order to emphasize the inclusion of (implicit) tadpole contributions in the FJ scheme. Here, the superscript "Pr" stands for renormalization prescriptions such as using the $\overline{\mathrm{MS}}$ or OS scheme, which needs to be specified for discussing the gauge dependence of finite parts. Concerning the latter, one finds that the FJ scheme also leads to gauge-independent one-loop mass parameter definitions in the OS scheme.

There is a simple explanation of why the reintroduction of tadpoles in the FJ scheme gives a gauge-independent result. As an example, we consider a fermion one-loop pole mass in a bare perturbation theory:

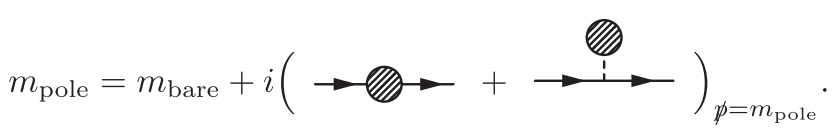

The whole expression Eq. (33) is gauge independent, as it is a one-loop pole mass [27], while $m_{\text {bare }}$ is gauge independent by principle. Thus, the mass shift, i.e., the term in parentheses in Eq. (33), is gauge independent as well. This means that the gauge dependence of the tadpole contributions induced by the Goldstone boson tadpoles of Eq. (16) is canceled when added to the 1PI contributions. With the FJ scheme implicitly including the tadpole contributions, one realizes that the mass renormalization constants of Eq. (30) therefore are gauge independent in terms of their finite parts as well when defined in an OS scheme. Note that in the PRTS, where Eq. (15) enforces tadpoles to vanish in Eq. (33) and no VEV shift is introduced, $m_{\text {bare }}$ needs to compensate for the gauge dependence of the 1PI contributions for $m_{\text {pole }}$ to be gauge independent (see, e.g., [8]).

\section{Numerical comparison}

In this section, we want to show an explicit determination of the VEV-renormalization constants in the various schemes and give a numerical example for their comparison.

We can use the relations Eq. (21) to define the VEV counterterms via the renormalization constants $\delta g_{2}$ and $\delta m_{W}^{2}$ as

$$
\delta v(\{\delta p\})=\delta v\left(\delta g_{2}, \delta m_{W}^{2}\right)=v\left(\frac{\delta m_{W}^{2}}{2 m_{W}^{2}}-\frac{\delta g_{2}}{g_{2}}\right),
$$

which holds for both tadpole schemes. The $S U(2)$-coupling $g_{2}$ can in turn be expressed as

$$
\delta g_{2}=\frac{e}{2 s_{w}} \frac{\delta e+\delta m_{W}^{2}-c_{w}^{2} \delta m_{Z}^{2}}{m_{Z}^{2}-m_{W}^{2}} .
$$

This step is usually done such that physical quantities known to high accuracy can be used as input parameters. The charge renormalization constant $\delta e$ can be defined in terms of the $\gamma-\gamma$ self energy and the $\gamma-Z$ mixing [1]. It does not depend on the choice of a tadpole scheme (because the Higgs field $h$ does not couple to the photon, and there is no $\gamma-Z-h$ vertex) and neither does $\delta g_{2}$ as defined in Eq. (35). Instead, the difference between the VEV-renormalization constants comes about via the definition of $\delta m_{W}^{2}$. As explained in Sec. IVA, the FJ scheme is equivalent to including tadpoles in the counterterm definitions either implicitly as in Eq. (32), or explicitly as if they were not renormalized at all. This means we can define

$$
\left.\delta m_{W}^{2}\right|_{\mathrm{FJ}}=\operatorname{Re}\left[-i\left(\tilde{W}_{W}+\underset{W}{\tilde{W}_{W} \tilde{m}_{W}}\right)\right]_{p^{2}=m_{W}^{2}}^{\text {transverse }}
$$

for the on-shell $W$-boson mass counterterm. This definition is gauge independent. In contrast to that, no tadpole contributions enter Eq. (34) in the PRTS, leading to the definition

$$
\left.\delta m_{W}^{2}\right|_{\text {PRTS }}=\operatorname{Re}\left[-i\left(\tilde{W}_{W}\right)\right]_{p^{2}=m_{W}^{2}}^{\text {transverse }}
$$

which is a gauge-dependent quantity. Hence, using Eq. (36) or Eq. (37) in Eq. (34) defines the VEV counterterm in the FJ or the PRTS scheme, respectively. A third version of the VEV counterterm is the definition via the Higgs background-field renormalization $\delta_{\hat{h}}$ using Eq. (B9). One possible renormalization condition for the latter is 


$$
\left.\frac{\partial}{\partial p^{2}} \Sigma_{\hat{h} \hat{h}}^{R}\right|_{p^{2}=m_{h}^{2}}=0
$$

where $\sum_{\hat{h} \hat{h}}^{R}$ is the one-loop renormalized self-energy of the Higgs background field. Then, we can define

$$
\left.\delta v\right|_{\mathrm{BG}}=\left.\frac{v}{2} \delta_{\hat{h}}\right|_{\mathrm{OS}}
$$

A definition of the VEV counterterm in terms of field renormalization constants can be expected to be gauge dependent, and this is indeed the case.

Now, using Eq. (34) together with Eq. (36) for the FJ scheme and Eq. (37) the PRTS, one can verify by an explicit one-loop calculation that

$$
\left.\delta v\right|_{\mathrm{PRTS}}=\left.\delta v\right|_{\mathrm{FJ}}+\left.\Delta v \stackrel{\infty}{=} \delta v\right|_{\mathrm{BG}} .
$$

This serves as a consistency check of Eq. (29) together with Eq. (12). Note that the second equal sign only holds for the UV parts of the counterterms, which is to be expected, because there is no simple relation between Eq. (38) and the other renormalization conditions in terms of finite parts. Nevertheless, we think it is illuminating to see a direct comparison of three rather different approaches to tadpole and VEV renormalization.

Using the numerical input parameters of Appendix E, the expressions above yield

$$
\begin{aligned}
\left.\delta v_{\text {fin }}\right|_{\mathrm{PRTS}} & =10.155 \mathrm{GeV}, \\
\left.\delta v_{\text {fin }}\right|_{\mathrm{FJ}} & =-138.457 \mathrm{GeV}, \\
\Delta v_{\text {fin }} & =148.612 \mathrm{GeV}, \\
\left.\delta v_{\text {fin }}\right|_{\mathrm{BG}} & =15.2504 \mathrm{GeV} .
\end{aligned}
$$

With these quantities being renormalization scale dependent and all of them except for Eq. (41b) being gauge dependent, one cannot put too much of an interpretation on these values. Nevertheless, it allows us to discuss two interesting aspects.

First, we see that Eq. (41b) being defined OS and Eq. (41c) show a large cancellation when combined to give Eq. (41a), which we tested for a wide range of renormalization scale values. In theories where not all model parameters can be defined via process-independent physical observables in an OS scheme, such cancellations can be absent, potentially leading to sizable shifts when $\Delta$ is included in parameter definitions (for the sake of gauge independence).

Second, both the VEV shift as well as $\left.\delta v_{\text {fin }}\right|_{\mathrm{FJ}}$ receive their largest contribution from the heaviest SM particle, the top quark. While in the SM, one could minimize the numerical effect of $\Delta v$ or $\left.\delta v_{\text {fin }}\right|_{\mathrm{FJ}}$ by choosing a renormalization scale $\mu_{R}$ such that the top-quark tadpole vanishes, ${ }^{9}$ this would not be of help in theories with particles at much higher mass scales. This is because even if $\mu_{R}$ is chosen to minimize the effect of the heaviest particle tadpoles [i.e., via minimizing the effect of $\ln \left(\mu_{R}^{2} / m_{\text {heavy }}^{2}\right)$ in the resulting $A_{0}$ functions], the lighter ones would in turn yield sizable shifts due to the large-scale difference between $\mu_{R}$ and $m_{\text {light }}$.

These aspects are in accordance with the findings of [17] where it was shown that tadpole contributions can be a source of numerical instabilities. Another example is the multi-Higgs-doublet Standard Model discussed by one of the authors in [7], where a $\overline{\mathrm{MS}}$ scheme is used and heavy Majorana fermions give large contributions to $\Delta v$, ultimately leading to very large corrections for one-loop neutrino masses.

In this sense, the FJ scheme can present a trade-off between gauge-independent quantities and numerical stability in a perturbative calculation.

\section{Remarks}

(i) The considerations in Sec. IVA are helpful for adjusting parameter input values in one-loop calculations from the FJ scheme to ones without tadpole contributions. As an example, we mention the $\overline{\mathrm{MS}}$ scheme, where from Eq. (29) one finds

$$
\left.v^{\overline{\mathrm{MS}}}\right|_{\mathrm{FJ}}=v^{\overline{\mathrm{MS}}}\left(1-\Delta_{\text {finite }}\right),
$$

and similar relations for the masses via Eq. (30). In addition, since the lhs of Eq. (42) is gauge independent, one can fully account for the gauge dependences on the rhs via the Goldstone contributions of $\Delta$.

(ii) It is rather straightforward to generalize the procedure of Sec. IVA to BSM models with altered scalar sectors, and we refer to the existing presentations of $[1,8]$. The two-Higgs-doublet model is one example. Here, a simple way to generalize the FJ procedure is to choose the Higgs basis [49-51]. Then, one can straightforwardly use Eq. (28) with $\Delta$ being identified by Eq. (28). The only difference is that $T_{h}$ and $\delta t_{h}$ now represent the tadpole contributions and the tadpole counterterms of the extended scalar sector in the Higgs basis. Another example is the multi-Higgs-doublet model as discussed in [7], where one can attribute a FJ term $\Delta_{k}$ to each doublet $\phi_{k}$ and define these via the respective tadpole contributions $T_{k}$.

(iii) One can think about solving the issues of gauge dependence, numerical stability, and moreover, the

\footnotetext{
${ }^{9}$ To be precise, $\left.\delta v_{\text {fin }}\right|_{\mathrm{FJ}}$ vanishes at a scale of $\mu_{R}^{2} \simeq$ $(182 \mathrm{GeV})^{2} / \exp (1)$.
} 
compatibility of the background-field approach to VEV renormalization by investigating alternative definitions of $\Delta$. One alternative is to define

$$
\Delta=\frac{T_{h}^{(\xi)}}{v m_{h}^{2}},
$$

where $T_{h}^{(\xi)}$ are only the gauge-dependent parts of the tadpole contributions, i.e., the Goldstone boson tadpoles of Eq. (16). This could be justified by the claim that these contributions come about as an artifact of the global symmetry breaking effect of the $R_{\xi}$-gauge. In this approach, gauge independence as in the original FJ scheme would still be guaranteed, because the difference in the choices of $\Delta$ would only lie in gauge-independent contributions. Then, large contributions to $\Delta$, e.g., from the top quark or even heavier particles in BSM models, would be absent and lead to numerically smaller corrections. Nevertheless, one could argue that this choice of $\Delta$ seems somewhat arbitrary in the sense that not all tadpole contributions are treated on the same footing and that moreover, the extraction of the gaugedependent tadpole contributions might become less obvious at higher-loop orders.

(iv) As discussed in Sec. II, $\hat{\delta}_{\phi}$ absorbs the gaugedependent divergences coming from the introduction of the $R_{\xi}$-gauge. One could try to mimic the effect of a FJ-VEV shift via an appropriate choice of the finite parts of $\hat{\delta}_{\phi}$. An obvious attempt would be to use an OS condition for the renormalized two-point function of the physical Higgs field and the Higgs background field, ${ }^{10}$ i.e.,

$$
\left.\frac{\partial}{\partial p^{2}} \Sigma_{h h}\right|_{p^{2}=m_{h}^{2}}=0,\left.\quad \frac{\partial}{\partial p^{2}} \Sigma_{\hat{h} \hat{h}}\right|_{p^{2}=m_{h}^{2}}=0 .
$$

This yields

$$
\begin{aligned}
\left.\hat{\delta}_{\phi}\right|_{\text {OS }}= & \frac{1}{2}\left(\left.\delta_{h}\right|_{\mathrm{OS}}-\left.\delta_{\hat{h}}\right|_{\mathrm{OS}}\right) \\
= & \frac{1}{4} \xi g_{2}^{2} \frac{1}{(4 \pi)^{2}}\left[2 B_{0}\left(m_{h}^{2}, \xi m_{W}^{2}, \xi m_{W}^{2}\right)\right. \\
& \left.+\frac{1}{\cos ^{2} \theta_{W}} B_{0}\left(m_{h}^{2}, \xi m_{Z}^{2}, \xi m_{Z}^{2}\right)\right] .
\end{aligned}
$$

Remarkably, it gives the same functional expression as the unphysical Green's function used to check the divergences in Eq. (B11), except that the subtraction point is $p^{2}=m_{h}^{2}$ instead of $p^{2}=0$. This choice of

\footnotetext{
${ }^{10}$ This is in contrast to the approach of [9], where only infinite contributions are taken into account.
}

$\hat{\delta}_{\phi}$ is equivalent to using Eq. (39). The numerical comparison in Eq. (41) shows no coincidence with the other VEV renormalizations and therefore indicates that the renormalization conditions used in the PRTS are incompatible with Eq. (45).

In Appendix $\mathrm{C}$, we show that when promoting Eq. (17) to a condition on finite terms as well, so that $\hat{\delta}_{\phi}$ absorbs the full Goldstone tadpoles, it leads to a gauge-dependent charge renormalization condition. This setting is practically equivalent to using $\left.\hat{\delta}_{\phi}\right|_{\text {os }}$, apart from subtraction point being $p^{2}=0$ instead of $p^{2}=m_{h}^{2}$. Hence, it shows the incompatibility of $\left.\hat{\delta}_{\phi}\right|_{\text {OS }}$ with the usual charge renormalization condition as discussed in Sec. IV C.

In general, there seems to be no obvious way to define the finite parts of $\hat{\delta}_{\phi}$ in order to mimic the effect of the FJ procedure, and it therefore remains purely as a tool for studying divergence structures.

\section{CONCLUSIONS}

The symmetry breaking effect of the $R_{\xi}$-gauge fixing leads to the necessity of a renormalization constant in addition to the ones for parameters and fields for the option to render all $n$-point Green's functions finite. By adapting the findings of [9] to the SM, we showed explicitly how this independent renormalization constant is related to the Higgs background-field renormalization and to Goldstone boson tadpoles. Effectively, this degree of freedom was used in the tadpole condition already in [40], yet we wanted to emphasize its origin lying in the gauge fixing as opposed to being a direct consequence of spontaneous symmetry breaking. We showed how this degree of freedom leads to gauge dependences in all the counterterms it enters, such as the mass counterterms in Table I.

The FJ scheme [16] manages to avoid these gauge dependences in parameter and counterterm definitions. The scheme was originally presented with arguments about using the proper VEV, while in the recent literature, the notion of true one-loop minimum [1] was employed. We, however, showed that one can look at this scheme as being simply a set of convenient counterterm redefinitions and in this way provide some independence from the interpretation of these notions.

The global symmetry argument, which lets us claim that we need only one additional renormalization constant $\hat{\delta}_{\phi}$, breaks down whenever $\xi_{W} \neq \xi_{Z}$. On the other hand, the FJ scheme generalizes to any loop order straightforwardly also when $\xi_{W} \neq \xi_{Z}$, even though it implicitly uses the degree of freedom of the Higgs background-field renormalization. This hints at the possibility that a single renormalization constant $\hat{\delta}_{\phi}$ is enough also in this case, yet it is unclear whether there exists a rigorous symmetry argument for that. 
Hence, there is a subtle interplay between the VEV renormalization interpreted as the renormalization of the Higgs background field, and the FJ scheme.

We used the SM as a playground to test various aspects of the renormalization in the $R_{\xi}$-gauge with a special emphasis on tadpole conditions and the connection between different approaches to the subject. This becomes especially relevant in BSM models with extended scalar sectors, where, e.g., numerical effects of tadpole contributions and the discussion of gauge dependences in mixing angles remain actively discussed $[17,52]$. We advocate the use of the FJ prescription for keeping track of gauge dependences in intermediate expressions and as a useful tool for consistency checks in perturbative calculations. Nevertheless, we also draw attention to settings where the FJ scheme can yield large corrections in renormalized quantities, potentially leading to numerical instabilities in non-OS schemes and discuss a possible alternative.

\section{ACKNOWLEDGMENTS}

The authors are grateful to Thomas Gajdosik for discussions and a careful reading of the manuscript. We would also like thank João P. Silva and Dominik Stöckinger for encouraging us to carry out this project. M. L. also thanks Marcel Krause and Stefan Liebler for many helpful discussions. V. D. thanks the Lithuanian Academy of Sciences for their support via Project No. DaFi2019. M. L. is supported partially by the DFG Collaborative Research Center TRR 257 "Particle Physics Phenomenology after the Higgs Discovery."

\section{APPENDIX A: GAUGE-FIXING FUNCTIONS}

In the background-field formalism, the gauge-fixing functions for the $U(1)$ and $S U(2)$ gauge-fixing parts, respectively, are given by

$$
\begin{gathered}
F_{B}=\partial^{\mu} B_{\mu}-i \xi g_{1}\left(\hat{\phi}^{\dagger} \frac{Y}{2} \phi-\phi^{\dagger} \frac{Y}{2} \hat{\phi}\right), \\
F_{W}^{i}=\partial^{\mu} W_{\mu}^{i}-i \xi g_{2}\left(\hat{\phi}^{\dagger} \frac{\sigma^{i}}{2} \phi-\phi^{\dagger} \frac{\sigma^{i}}{2} \hat{\phi}\right),
\end{gathered}
$$

where $\phi$ is the Higgs doublet field, and $\hat{\phi}$ is its background field. Both fields $\phi$ and $\hat{\phi}$ transform in the same way under the global gauge transformation; hence, it is easy to show that Eq. (A1) is invariant under the gauge transformation, while $F_{W}^{i}$ of Eq. (A2) transforms as a vector in the adjoint representation of $S U(2)$. The antighost $\bar{c}^{i}$ also transforms as a vector in the adjoint representation; hence, the gaugefixing term

$$
\mathcal{L}_{G F}=s\left[\bar{c}^{i}\left(F^{i}+\frac{\xi}{2} B^{i}\right)\right]
$$

is invariant. The mass eigenstate gauge-fixing functions are recovered by

$$
\begin{gathered}
F_{A}=F_{W}^{3} s_{W}+F_{B} c_{W}, \\
F_{Z}=F_{W}^{3} c_{W}-F_{B} s_{W}, \\
F_{W^{ \pm}}=\frac{1}{\sqrt{2}}\left(F^{1} \mp i F^{2}\right),
\end{gathered}
$$

where $s_{W}$ and $c_{W}$ are the sine and cosine of the Weinberg angle, respectively. Taking the limit

$$
\hat{\phi} \rightarrow\left(\begin{array}{c}
0 \\
\frac{1}{\sqrt{2}} v
\end{array}\right)
$$

in Eqs. (A1) and(A2) and inserting them into Eqs. (A4)(A6), we recover the usual $R_{\xi}$-gauge-fixing functions:

$$
\begin{aligned}
& F_{A}=\partial^{\mu} A_{\mu}, \\
& F_{Z}=\partial^{\mu} Z_{\mu}-\xi m_{Z} G_{Z}, \\
& F_{W}^{ \pm}=\partial^{\mu} W_{\mu}^{ \pm} \mp i \xi m_{W} G_{W}^{ \pm} .
\end{aligned}
$$

\section{APPENDIX B: CALCULATION USING BRST SOURCES}

Following [9], we find the divergence structure of $\hat{\delta}_{\phi}$ directly from the unphysical Green's functions that include BRST sources. The Higgs doublet of the SM is decomposed into quantum and background field:

$$
\Phi=\phi+\hat{\phi} .
$$

The fields $\Phi$ and $\hat{\phi}$ correspond to $\phi^{\text {eff }}$ and $\hat{\phi}+\hat{v}$ of [9], respectively. The BRST transformation of the background field is postulated to be in a contractible pair with another background field $\hat{q}$,

$$
s \hat{\phi}=\hat{q}_{\phi}, \quad s \hat{q}_{\phi}=0,
$$

so neither $\hat{\phi}$ nor $\hat{q}$ contribute to the BRST cohomology [53]. In other words, they do not contribute to the physical spectrum of the theory. The BRST transformation for the field $\Phi$ is

$$
s \Phi=g_{2} i \frac{\sigma^{k}}{2}(\phi+\hat{\phi}) c_{k}+\frac{i}{2} g_{1}(\phi+\hat{\phi}) c_{B} .
$$

From Eqs. (B2) and (B3), we get

$s \phi=\left(g_{2} i \frac{\sigma^{k}}{2}(\phi+\hat{\phi}) c_{k}+\frac{i}{2} g_{1}(\phi+\hat{\phi}) c_{B}-\hat{q}_{\phi}\right)$, 
where $\sigma$ are Pauli matrices, and $c_{k}$ and $c_{B}$ are the ghost fields of the $S U(2)$ and $U(1)$ gauge group, respectively. Finally, we include the BRST source $K_{\phi}$ in the Lagrangian

$$
\mathcal{L}_{K}=K_{\phi}^{\dagger} s \phi+s \phi^{\dagger} K_{\phi}
$$

The renormalization transformations of the field and background field from Eq. (8) are

$$
\phi \rightarrow \sqrt{\bar{Z}_{\phi} / \hat{Z}_{\phi}} \phi, \quad \hat{\phi} \rightarrow \sqrt{\bar{Z}_{\phi} \hat{Z}_{\phi}} \hat{\phi}
$$

thus, the introduced "technical" background field $\hat{q}$ transform as

$s \hat{\phi} \rightarrow s \sqrt{\bar{Z}_{\phi} \hat{Z}_{\phi}} \hat{\phi}=\sqrt{\bar{Z}_{\phi} \hat{Z}_{\phi}} \hat{q} \Rightarrow \hat{q} \rightarrow \sqrt{\bar{Z}_{\phi} \hat{Z}_{\phi}} \hat{q}$.

BRST sources transform as the inverse renormalization transformation of the corresponding field. Then, the relation

$$
\frac{\delta \Gamma}{\delta K_{\phi}}=\langle s \phi\rangle,
$$

where $\Gamma$ is the effective vertex functional and is unchanged after the renormalization transformation. From Eq. (B6), we get that the transformation for the BRST source of the Higgs doublet quantum field is

$$
K_{\phi} \rightarrow \sqrt{\hat{Z}_{\phi} / \bar{Z}_{\phi}} K_{\phi}
$$

Including all these renormalization transformations into Eq. (B5), we get

$$
\begin{aligned}
\mathcal{L}_{K}= & K_{\phi}^{\dagger}\left(g_{2} i \frac{\sigma^{k}}{2}\left(\phi+\hat{Z}_{\phi} \hat{\phi}\right) c_{k}+\frac{i}{2} g_{1}\left(\phi+\hat{Z}_{\phi} \hat{\phi}\right) c_{B}\right) \\
& -\hat{Z}_{\phi} K_{\phi}^{\dagger} \hat{q}_{\phi}+\text { H.c. }
\end{aligned}
$$

The last term gives the counterterm for an unphysical Green's function that includes only $\hat{Z}_{\phi}$. We will look at the Green's function $\Gamma_{\hat{q}_{h} K_{h}}$, where $h$ is the Higgs field component of the doublet. The counterterm of this Green's function is shown in the last diagram of Fig. 1. To calculate this Green's function at one loop, one only needs the Feynman rules for interactions between $\hat{q}, K_{\phi}$, and $c$, which can be read out from Eqs. (B10) and (A3), and are shown in Fig. 1. The loop diagram that we will need to calculate is shown in Fig. 2. The result of the sum of Fig. 2 and the last diagram of Fig. 1 is
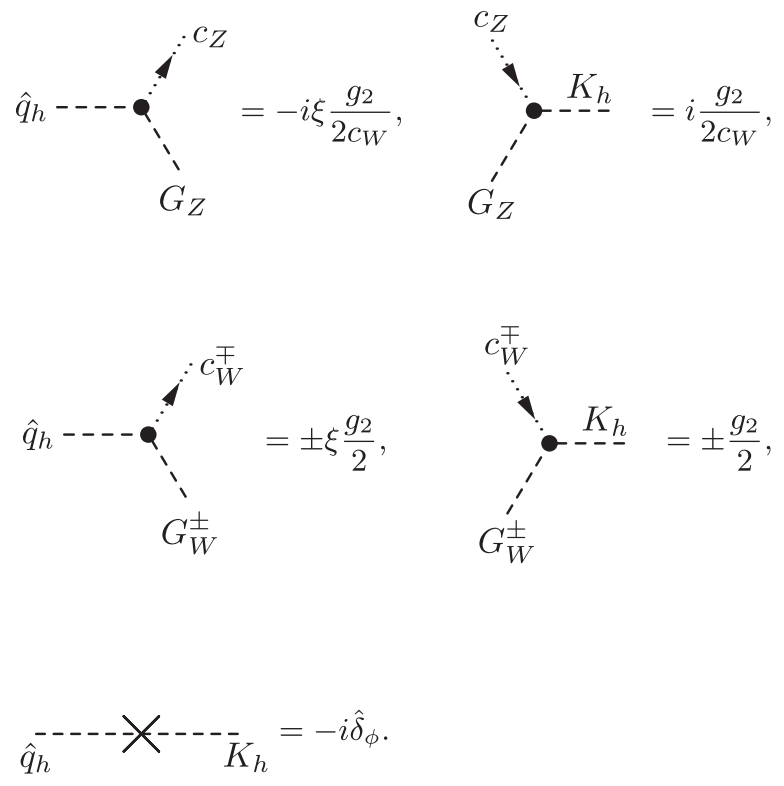

FIG. 1. Feynman rules for calculating $\hat{\delta}_{\phi}$.

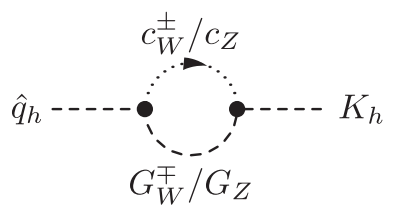

FIG. 2. One-loop diagram for calculating $\hat{\delta}_{\phi}$.

$$
\begin{aligned}
i \hat{\Gamma}_{\hat{q}_{h} K_{h}}^{[1]}= & -i \hat{\delta}_{\phi}+i \frac{1}{4} \xi g_{2}^{2} \frac{1}{(4 \pi)^{2}}\left[2 B_{0}\left(0, \xi m_{W}^{2}, \xi m_{W}^{2}\right)\right. \\
& \left.+\frac{1}{\cos ^{2} \theta_{W}} B_{0}\left(0, \xi m_{Z}^{2}, \xi m_{Z}^{2}\right)\right],
\end{aligned}
$$

or, using $A_{0}\left(m^{2}\right)=m^{2}\left(1+B_{0}\left(0, m^{2}, m^{2}\right)\right), m_{W}=\frac{g_{2} v}{2}$, and $m_{W}=\cos \theta_{W} m_{Z}$ :

$$
\begin{aligned}
\hat{\Gamma}_{\hat{q}_{h} K_{h}}^{[1]}= & -\hat{\delta}_{\phi}+\frac{1}{(4 \pi)^{2} v^{2}}\left\{2\left[A_{0}\left(m_{W}^{2} \xi\right)-\xi m_{W}^{2}\right]\right. \\
& \left.+\left[A_{0}\left(m_{Z}^{2} \xi\right)-\xi m_{Z}^{2}\right]\right\} .
\end{aligned}
$$

The finiteness of this two-point function fixes the divergences of $\hat{\delta}_{\phi}$. Moreover, Eq. (B12) immediately shows that its divergences coincide with the ones of the Goldstone boson tadpoles Eq. (16), which means that $\hat{\delta}_{\phi}$ indeed absorbs all the gauge-dependent divergences in the tadpole condition Eq. (15).

\section{APPENDIX C: GAUGE DEPENDENCE IN THE BACKGROUND-FIELD-MODIFIED OS SCHEME}

In this section, we present the consequence of promoting Eq. (17) to a renormalization condition on finite parts, namely, 


$$
T_{h}^{(\xi)}-\lambda v^{3} \hat{\delta}_{\phi}=0
$$

In principle, this is a valid renormalization condition, which allows us to absorb all the tadpole finite gauge dependences into $\hat{\delta}_{\phi}$. One can verify that the FJ term $\Delta$ is

$$
\Delta=\frac{1}{2} \hat{\delta}_{\phi}+\text { gauge independent }
$$

when Eq. (C1) holds. This allows for a more direct interpretation of a FJ term in the sense of a backgroundfield renormalization also in finite parts, leading to a gauge-independent OS mass renormalization constant. Also, the form of Eq. (C2) gives a possibility to modify the FJ procedure to include only the gauge-dependent term, namely, $\hat{\delta}_{\phi}$. However, we will show that this choice, together with the OS conditions on the two-point functions, leads to a gauge-dependent charge renormalization constant and cannot be used with the usual charge renormalization condition presented in, e.g., $[1,32,33]$.

To show this, we first need to get the gauge-dependent part of $\bar{\delta}_{\phi}$ in this scheme. For that, we look at the gaugedependent part of the Higgs self-energy, which can be written as

$$
\begin{aligned}
\Sigma_{\xi}= & \frac{1}{(4 \pi v)^{2}}\left(p^{2}-m_{h}^{2}\right)\left(f_{Z}+2 f_{W}\right) \\
& +m_{h}^{2} \frac{1}{(4 \pi v)^{2}} \frac{3}{2}\left(A_{0}\left(m_{Z}^{2} \xi_{Z}\right)+2 A_{0}\left(m_{W}^{2} \xi_{W}\right)\right) \\
f_{V}= & A_{0}\left(m_{V}^{2} \xi\right)-\frac{1}{2}\left(p^{2}+m_{h}^{2}\right) B_{0}\left(p^{2}, m_{V}^{2} \xi, m_{V}^{2} \xi\right) .
\end{aligned}
$$

The gauge-dependent part of the renormalized one-loop self energy function is

$$
\Sigma_{\xi}^{R}=\left.\delta_{h}\right|_{\xi} p^{2}-\left.\left(\delta_{m_{h}^{2}}+\delta_{h}\right)\right|_{\xi} m_{h}^{2}+\Sigma_{\xi},
$$

where we write $\left.\right|_{\xi}$ to denote the gauge-dependent terms of the renormalization constants. The OS conditions give

$$
\left.\frac{\partial}{\partial p^{2}} \Sigma^{R}\right|_{p^{2}=m_{H}^{2}}=0,\left.\quad \Sigma^{R}\right|_{p^{2}=m_{H}^{2}}=0 .
$$

Inserting Eqs. (C3)-(C5) into OS conditions Eq. (C6) to check the gauge-dependent parts, we get the gauge dependences of the mass and field renormalization constants of the OS scheme (i.e., in the tadpole scheme of Ref. [32]):

$$
\left.\delta_{m_{h}^{2}}\right|_{\xi}=\frac{1}{(4 \pi v)^{2}} \frac{3}{2}\left[A_{0}\left(m_{Z}^{2} \xi_{Z}\right)+2 A_{0}\left(m_{W}^{2} \xi_{W}\right)\right],
$$

$$
\begin{aligned}
\left.\delta_{h}\right|_{\xi}= & -\frac{1}{(4 \pi v)^{2}}\left\{\left[A_{0}\left(m_{Z}^{2} \xi\right)+2 A_{0}\left(m_{W}^{2} \xi\right)\right]\right. \\
& \left.-m_{h}^{2}\left[B_{0}\left(p^{2}, m_{Z}^{2} \xi, m_{Z}^{2} \xi\right)+2 B_{0}\left(p^{2}, m_{W}^{2} \xi, m_{W}^{2} \xi\right)\right]\right\} .
\end{aligned}
$$

By using the expression for $\Delta$ from Eq. (C2) and $\hat{\delta}_{\phi}$, fixed by Eq. (C1), we see that the FJ-OS mass counterterm, as defined in Eq. (30c), is truly gauge independent:

$$
\left.\left(\left.\delta_{m_{h}^{2}}\right|_{F J}\right)\right|_{\xi}=\left.\delta_{m_{h}^{2}}\right|_{\xi}-3 \Delta_{\xi}=\left.\delta_{m_{h}^{2}}\right|_{\xi}-\frac{3}{2} \hat{\delta}_{\phi}=0 .
$$

From Eq. (12), we can get the field renormalization part $\bar{\delta}_{\phi}$ that does not have gauge-dependent divergences. However, it turns out that in the OS, the finite part of $\bar{\delta}_{\phi}$ is gauge dependent:

$$
\begin{aligned}
\left.\bar{\delta}_{\phi}\right|_{\xi}= & \left.\delta_{h}\right|_{\xi}+\hat{\delta}_{\phi} \\
= & \frac{1}{(4 \pi v)^{2}} m_{h}^{2}\left[B_{0}\left(p^{2}, m_{Z}^{2} \xi, m_{Z}^{2} \xi\right)\right. \\
& \left.+2 B_{0}\left(p^{2}, m_{W}^{2} \xi, m_{W}^{2} \xi\right)\right] .
\end{aligned}
$$

Note that the divergences in this term are gauge independent as they should be.

Now using Eq. (C1) together with Eq. (28), we see that we must have

$$
\left.\delta_{\lambda}\right|_{\xi}+\left.\bar{\delta}_{\phi}\right|_{\xi}-\left.\delta_{\mu^{2}}\right|_{\xi}=0 .
$$

From the fact that the FJ mass renormalization constant of the Higgs boson coincides with $\delta_{\mu^{2}}$ (see Table I) and is gauge independent, the gauge dependence of $\delta_{\lambda}$ is

$$
\left.\delta_{\lambda}\right|_{\xi}=-\left.\bar{\delta}_{\phi}\right|_{\xi}
$$

From Table I, we see that $\delta_{\lambda}$ enters the definition of the FJ mass renormalization constants, which are gauge independent. Hence, from the gauge independence of $\left.\delta_{M_{W}^{2}}\right|_{F J}$, $\left.\delta_{M_{Z}^{2}}\right|_{\mathrm{FJ}}$ and $\left.\delta_{m_{f}}\right|_{\mathrm{FJ}}$, we get

$$
\begin{gathered}
0=\left.2 \delta_{g_{1,2}}\right|_{\xi}-\left.\delta_{\lambda}\right|_{\xi}=\left.2 \delta_{g_{1,2}}\right|_{\xi}+\left.\bar{\delta}_{\phi}\right|_{\xi}, \\
0=\left.\delta_{y}\right|_{\xi}-\left.\frac{1}{2} \delta_{\lambda}\right|_{\xi}=\left.\delta_{y}\right|_{\xi}+\left.\frac{1}{2} \bar{\delta}_{\phi}\right|_{\xi},
\end{gathered}
$$

which leads to

$$
\frac{1}{g_{1}^{2}+g_{2}^{2}}\left(\left.g_{1}^{2} \delta_{g_{2}}\right|_{\xi}+\left.g_{2}^{2} \delta_{g_{1}}\right|_{\xi}\right)+\left.\bar{\delta}_{\phi}\right|_{\xi}=\left.\delta_{e}\right|_{\xi}+\left.\bar{\delta}_{\phi}\right|_{\xi}=0 .
$$


From Eq. (C15), we see that the charge renormalization constant $\delta_{e}$ is gauge dependent, because it needs to cancel the gauge dependence of $\left.\bar{\delta}_{\phi}\right|_{\xi}$ given in Eq. (C10). Note that this is solely because we enforced Eq. (C1). Thus, we have two tadpole conditions, pole and residue conditions for the $W, Z$, and Higgs two-point function, which are in total eight conditions; hence, they fully determine $g_{1,2}, \delta_{\lambda}, \delta_{\mu^{2}}$, $\bar{\delta}_{\phi}, \hat{\delta}_{\phi}$, and the field renormalization constants of the $Z$ and $W$ bosons. This means that there is no freedom left to impose a charge renormalization condition as in, e.g., $[1,32,33]$, which would give a gauge-independent charge renormalization constant otherwise. Nevertheless, it is interesting to see that the gauge-independent definition of the charge renormalization constant is possible also in this "scheme" by absorbing $\bar{\delta}_{\phi}$ into their definitions, as suggested by Eq. (C15). Hence, in principle, it is possible to use Eq. (C1) instead of the usual charge renormalization condition and even define a gauge-independent charge renormalization constant. Yet to understand all the consequences of this unconventional choice, a more thorough study is needed which is beyond the scope of this work.

\section{APPENDIX D: EXPLICIT DIVERGENCES}

We used the SM file from FeynArts together with FormCalc to get the explicit expressions for the divergences. The divergences of the one-, two-, three-, and four-point function of the Higgs boson in the SM, respectively, are

$$
\begin{gathered}
\Gamma_{h}^{\mathrm{UV}}=v^{3}(A+G+S), \\
\Gamma_{h h}^{\mathrm{UV}}=p^{2}\left(\frac{1}{v^{2}} B+\frac{2}{m_{h}^{2}} G\right)+v^{2}(3 A+5 S+G), \\
\Gamma_{h h h}^{\mathrm{UV}}=6 v(A+2 S-G), \\
\Gamma_{h h h h}^{\mathrm{UV}}=6(A+2 S-2 G),
\end{gathered}
$$

where we abbreviated

$$
\begin{gathered}
A=-\frac{1}{\left(4 \pi v^{2}\right)^{2}}\left[4\left(\sum_{f=e, \mu, \tau} m_{f}^{4}+3 \sum_{q=u, d, s, c, t, b} m_{q}^{4}\right)\right. \\
\left.-3\left(2 m_{W}^{4}+m_{Z}^{4}\right)\right], \\
B=\frac{1}{(4 \pi v)^{2}}\left[2\left(\sum_{f=e, \mu, \tau} m_{f}^{2}+3 \sum_{q=u, d, s, c, t, b} m_{q}^{2}\right)\right. \\
\left.-3\left(2 m_{W}^{2}+m_{Z}^{2}\right)\right], \\
S=\frac{3}{2} \frac{m_{h}^{4}}{\left(4 \pi v^{2}\right)^{2}}, \quad G=\frac{1}{2} \frac{m_{h}^{2}}{\left(4 \pi v^{2}\right)^{2}}\left(2 m_{W}^{2} \xi+m_{Z}^{2} \xi\right),
\end{gathered}
$$

and omitted a global factor of $\frac{2}{4-D}$. The divergences in $A$ and $B$ come from loop diagrams with ghosts, vectors, and fermions. Note that neither $A$ nor $B$ are gauge dependent, since ghost and vector boson gauge dependences cancel exactly. The divergences abbreviated as $S$ come from diagrams with the Higgs boson loop contribution. Finally, the only gauge-dependent UV divergent term $G$ corresponds to divergences of the Goldstone boson loop and vanishes in the case of $\xi \rightarrow 0$. Note that we wrote all these functions in terms of four abbreviated constants; thus, we need four independent conditions and 4 degrees of freedom to uniquely fix them. In the case of $\xi \rightarrow 0$, the number is reduced to only 3 . The 4 degrees of freedom are $\mu, \lambda, \bar{\delta}_{\phi}$, and $\hat{\delta}_{\phi}$. They appear in the counterterms of one-, two-, three-, and four-point functions of the Higgs boson, respectively,

$$
\delta \Gamma_{h}=-\frac{1}{2} m_{h}^{2} v\left(\delta_{\lambda}-\delta_{\mu^{2}}+\bar{\delta}_{\phi}+\hat{\delta}_{\phi}\right),
$$

$\delta \Gamma_{h h}=-\frac{1}{2} m_{h}^{2}\left(3 \delta_{\lambda}-\delta_{\mu^{2}}+5 \bar{\delta}_{\phi}+\hat{\delta}_{\phi}\right)+p^{2}\left(\bar{\delta}_{\phi}-\hat{\delta}_{\phi}\right)$,

$$
\begin{gathered}
\delta \Gamma_{h h h}=-3 \frac{m_{h}^{2}}{v}\left(\delta_{\lambda}+2 \bar{\delta}_{\phi}-\hat{\delta}_{\phi}\right), \\
\delta \Gamma_{h h h h}=-3 \frac{m_{h}^{2}}{v^{2}}\left(\delta_{\lambda}+2 \bar{\delta}_{\phi}-2 \hat{\delta}_{\phi}\right) .
\end{gathered}
$$

To make sure that the renormalized $n$-point functions are finite, we solve four equations:

$$
\begin{gathered}
\delta \Gamma_{h}^{\mathrm{UV}}+\Gamma_{h}^{\mathrm{UV}}=0, \\
\delta \Gamma_{h h}^{\mathrm{UV}}+\Gamma_{h h}^{\mathrm{UV}}=0, \\
\frac{\partial}{\partial p^{2}}\left(\delta \Gamma_{h h}^{\mathrm{UV}}+\Gamma_{h h}^{\mathrm{UV}}\right)=0, \\
\delta \Gamma_{h h h}^{\mathrm{UV}}+\Gamma_{h h h}^{\mathrm{UV}}=0 .
\end{gathered}
$$

They give us

$$
\begin{gathered}
\delta_{\mu^{2}}=B+\frac{1}{\lambda} S, \quad \delta_{\lambda}=2\left(B+\frac{1}{\lambda} S\right)+\frac{1}{\lambda} A, \\
\hat{\delta}_{\phi}=\frac{1}{\lambda} G, \quad \bar{\delta}_{\phi}=-B, \quad \lambda=\frac{m_{h}^{2}}{2 v^{2}} .
\end{gathered}
$$

Inserting these expressions into Eq. (D11), we automatically get

$$
\delta \Gamma_{h h h h}^{\mathrm{UV}}+\Gamma_{h h h h}^{\mathrm{UV}}=0,
$$

where $\Gamma_{h h h h}^{\mathrm{UV}}$ is given in Eq. (D4).

The VEV counterterms presented in Sec. IV C have divergences, which can be read out from Table I, inserting the expressions for the renormalization constants shown in Eq. (D16):

$$
\delta v=\frac{1}{2} v\left(\hat{\delta}_{\phi}+\bar{\delta}_{\phi}\right)=\frac{1}{2} v\left(\frac{1}{\lambda} G-B\right),
$$




$$
\begin{aligned}
\left.\delta v\right|_{\mathrm{FJ}} & =\frac{1}{2} v\left(\delta_{\mu^{2}}-\delta_{\lambda}\right)=-\frac{1}{2} v\left(B+\frac{1}{\lambda} S+\frac{1}{\lambda} A\right), \\
\left.\delta v\right|_{\mathrm{FJ}} & =\delta v+\Delta v \\
& \Rightarrow \Delta v=-\frac{1}{2} v\left(\frac{1}{\lambda} S+\frac{1}{\lambda} A+\frac{1}{\lambda} G\right)=-\frac{1}{m_{h}^{2}} \Gamma_{h}^{\mathrm{UV}} .
\end{aligned}
$$

\section{APPENDIX E: INPUT VALUES}

Here we present the numerical input values used in Eqs. (41a)-(41d) together with the software package
LoopTools [54] at its standard renormalization scale of $\mu_{R}=1 \mathrm{GeV}$,

$\xi=1, \quad m_{W}=80.398 \mathrm{GeV}, \quad m_{Z}=91.1876 \mathrm{GeV}$,

$m_{h}=125.09 \mathrm{GeV}, \quad v=246.221 \mathrm{GeV}$,

$m_{e}=0.000510999 \mathrm{GeV}, \quad m_{\mu}=0.105658 \mathrm{GeV}$,

$m_{\tau}=1.77684 \mathrm{GeV}$,

$m_{u}=0.19 \mathrm{GeV}, \quad m_{c}=1.4 \mathrm{GeV}, \quad m_{t}=172.500 \mathrm{GeV}$,

$m_{d}=0.19 \mathrm{GeV}, \quad m_{s}=0.19 \mathrm{GeV}, \quad m_{b}=4.75 \mathrm{GeV}$,

$e=0.308147$.
[1] A. Denner and S. Dittmaier, Phys. Rep. 864, 1 (2020).

[2] P. A. Grassi, B. A. Kniehl, and A. Sirlin, Phys. Rev. D 65, 085001 (2002).

[3] S. Weinberg, Phys. Rev. D 7, 2887 (1973).

[4] S. Actis, A. Ferroglia, M. Passera, and G. Passarino, Nucl. Phys. B777, 1 (2007).

[5] M. Krause, R. Lorenz, M. Muhlleitner, R. Santos, and H. Ziesche, J. High Energy Phys. 09 (2016) 143.

[6] V. Dūdènas and T. Gajdosik, Phys. Rev. D 98, 035034 (2018).

[7] W. Grimus and M. Löschner, J. High Energy Phys. 11 (2018) 087.

[8] A. Denner, L. Jenniches, J.-N. Lang, and C. Sturm, J. High Energy Phys. 09 (2016) 115.

[9] M. Sperling, D. Stöckinger, and A. Voigt, J. High Energy Phys. 07 (2013) 132.

[10] M. Sperling, D. Stöckinger, and A. Voigt, J. High Energy Phys. 01 (2014) 068.

[11] T. Appelquist, J. Carazzone, T. Goldman, and H. R. Quinn, Phys. Rev. D 8, 1747 (1973).

[12] P. H. Chankowski, S. Pokorski, and J. Rosiek, Phys. Lett. B 274, 191 (1992).

[13] B. W. Lee and J. Zinn-Justin, Phys. Rev. D 5, 3121 (1972).

[14] B. W. Lee and J. Zinn-Justin, Phys. Rev. D 5, 3137 (1972); 8, 4654(E) (1973).

[15] B. W. Lee and J. Zinn-Justin, Phys. Rev. D 5, 3155 (1972).

[16] J. Fleischer and F. Jegerlehner, Phys. Rev. D 23, 2001 (1981).

[17] A. Freitas and D. Stöckinger, in Proceedings of the 10th International Conference on Supersymmetry and Unification of Fundamental Interactions (SUSY02) (DESY, Hamburg, Germany, 2002), pp. 657-661 [arXiv:hep-ph/0210372].

[18] M. Krause, Master's thesis, Karlsruhe Institute of Technology, Institute for Theoretical Physics, (2016).

[19] T. Hahn, Comput. Phys. Commun. 140, 418 (2001).

[20] R. Mertig, M. Böhm, and A. Denner, Comput. Phys. Commun. 64, 345 (1991).

[21] V. Shtabovenko, R. Mertig, and F. Orellana, Comput. Phys. Commun. 207, 432 (2016).
[22] V. Shtabovenko, R. Mertig, and F. Orellana, Comput. Phys. Commun. 256, 107478 (2020).

[23] T. Hahn and M. Perez-Victoria, Comput. Phys. Commun. 118, 153 (1999).

[24] T. Hahn, S. Paßehr, and C. Schappacher, Proc. Sci., LL2016 (2016) 068 [arXiv:1604.04611].

[25] A. Sirlin, Phys. Rev. Lett. 67, 2127 (1991).

[26] A. Denner, S. Dittmaier, M. Roth, and D. Wackeroth, Nucl. Phys. B560, 33 (1999).

[27] P. Gambino and P. A. Grassi, Phys. Rev. D 62, 076002 (2000).

[28] A. Denner, S. Dittmaier, M. Roth, and L. Wieders, Nucl. Phys. B724, 247 (2005).

[29] A. Denner and S. Dittmaier, Nucl. Phys. B, Proc. Suppl. 160, 22 (2006).

[30] B. A. Kniehl and A. Sirlin, Phys. Lett. B 530, 129 (2002).

[31] D. Espriu, J. Manzano, and P. Talavera, Phys. Rev. D 66, 076002 (2002).

[32] A. Denner, Fortschr. Phys. 41, 307 (1993).

[33] M. Böhm, A. Denner, and H. Joos, in Gauge Theories of Strong and Electroweak Interactions, 3rd ed. (B. G. Teubner, Stuttgart, 2001).

[34] O. Piguet and S. Sorella, Algebraic Renormalization: Perturbative Renormalization, Symmetries and Anomalies (Springer, Berlin, 1995), Vol. 28.

[35] R. Haussling and E. Kraus, Z. Phys. C 75, 739 (1997), arXiv:hep-th/9608160.

[36] E. Kraus, Ann. Phys. (N.Y.) 262, 155 (1998).

[37] E. Kraus and K. Sibold, Z. Phys. C 68, 331 (1995).

[38] E. Kraus and S. Groot Nibbelink, in Proceedings of the 4th National Summer School for German-Speaking Graduate Students of Theoretical Physics (1998) [arXiv:hep-th/ 9809069].

[39] E. Kraus, Acta Phys. Pol. B 29, 2647 (1998), https://www .actaphys.uj.edu.pl/fulltext?series=Reg\&vol=29\&page $=2647$.

[40] G. Passarino and M. J. G. Veltman, Nucl. Phys. B160, 151 (1979).

[41] C. Becchi, in Proceedings of the 2nd Triangle Graduate School in Particle Physics (1996) [arXiv:hep-ph/9705211]. 
[42] C. Itzykson and J. Zuber, Quantum Field Theory, International Series in Pure and Applied Physics (McGraw-Hill, New York, 1980).

[43] A. A. Slavnov, Theor. Math. Phys. 10, 99 (1972).

[44] N. K. Nielsen, Nucl. Phys. B101, 173 (1975).

[45] L. P. Alexander and A. Pilaftsis, J. Phys. G 36, 045006 (2009).

[46] H. H. Patel and M. J. Ramsey-Musolf, J. High Energy Phys. 07 (2011) 029.

[47] M. Garny and T. Konstandin, J. High Energy Phys. 07 (2012) 189.

[48] A. Denner, G. Weiglein, and S. Dittmaier, Nucl. Phys. B440, 95 (1995).
[49] S. Davidson and H. E. Haber, Phys. Rev. D 72, 035004 (2005); 72, 099902(E) (2005).

[50] H. E. Haber and D. O'Neil, Phys. Rev. D 74, 015018 (2006); 74, 059905(E) (2006).

[51] H. E. Haber and D. O’Neil, Phys. Rev. D 83, 055017 (2011).

[52] A. Denner, S. Dittmaier, and J.-N. Lang, J. High Energy Phys. 11 (2018) 104.

[53] G. Barnich, F. Brandt, and M. Henneaux, Phys. Rep. 338, 439 (2000).

[54] T. Hahn and M. Pérez-Victoria, Comput. Phys. Commun. 118, 153 (1999). 